\title{
Uso de las TIC en Arquitectura: experiencia de un programa tecnólogo de la Universidad del Atlántico, Barranquilla, Colombia
}

\section{Use of ICT in Architecture: experience of a technological program at the Universidad del Atlántico, Barranquilla, Colombia}

\author{
DOI: 10.17981/mod.arq.cuc.25.1.2020.03
}

Artículo. Fecha de Recepción: 09/04/2020. Fecha de Aceptación: 13/05/2020.

\author{
Cesar Martínez García (iD \\ Universidad del Atlántico. Barranquilla (Colombia) \\ atciltda@hotmail.com \\ Christiam Castro Escalante \\ Universidad del Atlántico. Barranquilla (Colombia) \\ cace78@hotmail.com \\ Delma Esther Rocha Álvarez \\ Universidad del Atlántico. Barranquilla (Colombia) \\ delmarocha@mail.uniatlantico.edu.co \\ Isaac Nieto Mendoza (iD \\ Universidad del Atlántico. Barranquilla (Colombia) \\ icnieto@mail.uniatlantico.edu.co
}

Para citar este artículo:

Martínez, C., Castro, C., Rocha, D. y Nieto, I. (2020). Uso de las TIC en Arquitectura: experiencia zde un programa tecnólogo de la Universidad del Atlántico, Barranquilla, Colombia. MODULO ARQUITECTURA CUC, (25), pp. 67-84, 2020. DOI: http://doi.org/10.17981/mod.arq.cuc.25.1.2020.03

\section{Resumen}

Analizar los procesos de enseñanza basados en el uso de las Tecnologías de la Información y Comunicaciones (TIC) de los docentes del programa Tecnología en Gestión de la Construcción de Proyectos Arquitectónicos y su incidencia en el desempeño académico y prácticas profesionales de los estudiantes en el semestre 2019-2 en la Universidad del Atlántico (sede Suán). Estudio de paradigma hermenéutico, enfoque cualitativo y de tipo descriptivo y documental. Se trabajó con docentes, estudiantes y egresados del programa Tecnología en Gestión de la Construcción de Proyectos Arquitectónicos, como también evaluadores de prácticas profesionales por medio de entrevista semiestructurada. El testimonio de los participantes dio cuenta de falencias en materia del uso de las TIC en el proceso de enseñanza/aprendizaje, que han incidido en el desempeño académico y en sus prácticas profesionales negativamente. Las dificultades en materia de uso de tic se asocian a la falta de acceso a dichos insumos tecnológicos, el manejo básico de herramientas de ofimática y softwares de uso común en el desarrollo de su carrera tecnóloga y en la vida laboral. Es preciso pensar que, para los modelos de enseñanza/aprendizaje, el uso de las TIC se hace necesario en aras de estimular el desarrollo cognitivo, fortaleciendo los procesos de formación e incidiendo asertivamente en el rendimiento académico de los estudiantes.

Palabras clave: Proceso de enseñanza; TIC; desempeño académico; prácticas profesionales

\section{Abstract}

Analyze the teaching processes based on the use of Information and Commu nication Technologies (ICT) of the teachers of the Technology in Construction Management of Architectural Projects program and its impact on the academic performance and professional practices of students in the 2019-2 semester at the Universidad del Atlántico (headquarters Suán). Study of hermeneutical paradigm qualitative approach and of a descriptive and documentary type. We worked with teachers, students and graduates of the Technology in Construction Management of Architectural Projects program, as well as evaluators of professional practices through a semi-structured interview. The testimony of the participants revealed shortcomings in the use of ICT in the teaching / learning process, which have negatively affected academic performance and professional practices. The difficulties in the use of ict are associated with the lack of access to these technological inputs, the basic use of office automation tools and softwares in common use in the development of their technological career and in working life. It is necessary to think that, for teaching / learning models, the use of ICT is necessary in order to stimulate cognitive development, strengthening training processes and assertively influencing the academic performance of students.

Keywords: Teaching process; ICT; academic performance; professional practices

(c) The author; licensee Universidad de la Costa - CUC. 


\section{INTRODUCCIÓN}

El análisis del proceso de enseñanza/aprendizaje en la formación en las IES tanto de técnico, tecnólogo y profesional, y de las repercusiones en el desempeño académico y laboral - en lo que respecta a la Educación Superior-, resulta fundamental en la calidad educativa y los aspectos relacionados con las competencias específicas en determinadas áreas del saber. Investigaciones en Educación, específicamente, las relacionadas con procesos de formación, reconocen la importancia de dicho análisis y el papel de la evaluación frecuente de las dinámicas educativas; conllevan a consensos académicos que brindan insumos al estudiante para un mejor desempeño de su profesión durante toda la vida (Pimienta, 2012; Guevara, 2013; Castillo y Del Castillo, 2015; Núñez, 2016; HernándezRojas, Suárez-Castrillón y Rico-Bautista, 2017; Hernández, 2017; Pérez, Daza y Orozco, 2017).

Desde la pedagogía, la enseñanza y el aprendizaje son procesos conjuntos, lo cual da cuenta de la inferencia de las estrategias docentes en los aprendizajes (Martínez, Tobón y Romero, 2017; Ruiz y Duarte, 2017; Martin, 2018), significando para la Educación Superior:

(...) la búsqueda de modelos educativos basados en las competencias y centrados en el estudiante, lo cual implica que dentro de sus prioridades está la búsqueda de nuevas formas de relación con la docencia, mucho más flexibles y creativas que potencien -desde la práctica- la resolución de problemas pedagógicos, considerando al estudiante como el actor principal en la construcción de su aprendizaje (León, Risco y Alarcón, 2014, p. 125).
No obstante, según Seguí, (citado en Botasso \& Tanoune, 2016), la formación de profesionales en el área de la arquitectura, en contextos ubicados en algunos países de América Latina "arrastra la dificultad de incorporar un ambiente reflexivo (teórico-práctico)" (p. 44), lo que ha conllevado a los formadores de arquitectura a no tener una postura pedagógica que les identifique; asimismo, la enseñanza en arquitectura, de acuerdo con Romero (2016), en países como Perú, ha estado arraigada a modelos de enseñanza tradicionalistas, donde las competencias educativas que soportan el programa académico, dejan de lado la integración de los avances tecnológicos dados por medio de la globalización y las nuevas formas de enseñanza.

De acuerdo con Castellanos, Sierra y Albis (2018), en Colombia, las condiciones mínimas de calidad de los programas de Educación Superior en Arquitectura, son reguladas por el Ministerio Nacional de Educación; no obstante, muchas instituciones no cuentan con los escenarios que garanticen la búsqueda de los énfasis y enfoques modernos, y la inducción en las problemáticas actuales de los contextos en el que el estudiante de arquitectura se desenvuelve, en aras de dar respuesta a las mismas (Cohen y Correa, 2018).

Es así que para Guevara (2013), el docente moderno en materia de arquitectura, preserva los pilares académicos establecidos desde los orígenes de la Educación Universitaria; donde los intereses institucionales no se encuentran 
anclados a que el estudiante esté en capacidad de crear e innovar, más bien, "se deseaba que reprodujeran su propia concepción, que era considerada como un modelo a imitar" (p. 2), valiéndose el aprendiz únicamente de dichas competencias para llevar a cabo su profesión.

Siguiendo entonces con la estructura de enseñanza en materia de arquitectura, en Educación Superior; dichas competencias resultan ser cimientos distantes — muchas veces- de los intereses del profesional actual. Es, entonces, como desde la educación, es preciso que se tenga en cuenta la relación de los componentes educativos con las realidades del mundo globalizado, brindando garantías tanto para su formación como su desempeño laboral (Vélez, 2011; Correal y Verdugo, 2011; Villar, 2012 como se citó en Guevara, 2013; Erazo y Sánchez, 2013; Rueda, 2014; Díaz, 2016; Gasco y Melo, 2017; O’Byrne y Quintana, 2018; Melo, 2018; Perlaza y Betancourt, 2018; Martínez y García, 2019; González, Portero, Machado y Fariñas, 2019).

En la investigación realizada por Hernández-Rojas et al. (2017), se reconoce la relevancia de adaptar nuevas alternativas en las competencias específicas de los programas de arquitectura, fundamentadas en la "incorporación de las Tecnologías de la Información y la Comunicación (TIC)" (Márquez, 2017, p. 21) fortaleciendo "el desarrollo de habilidades y destrezas en las prácticas docentes en la Educación Superior" (Hernández-Rojas et al., 2017, p. 129), afirmando que:
(...) este acercamiento temprano a las tecnologías, una constante comunicación y navegación en las redes con acceso casi ilimitado a la información que se puede encontrar en ella, facilita la inclusión de ciertas estrategias tecnológicas en los procesos de aprendizaje, generando una transformación cultural en la construcción de conocimiento donde de igual forma se produce un rol nuevo en el docente e inventando nuevos desafíos en el sector educativo (p. 126).

El reconocimiento e importancia de los procesos tecnológicos, permiten la reorientación de patrones educativos, induciendo a la inserción de metodologías activas e innovadoras que propicien el mejoramiento continuo de la calidad de la Educación Superior. Sin embargo, de acuerdo con el estudio realizado por Marín, Inciarte, Hernández y Pitre (2017), los entornos universitarios del departamento del Atlántico, a pesar de destacar la influencia de las TIC, el 71\% de las instituciones de Educación Superior "no disponen de cursos de actualización para que los docentes se capaciten en las TIC de manera continua" (Marín et al., 2017, p. 35), a la vez que, "el 64\% de las instituciones de Educación Superior estudiadas en la ciudad de Barranquilla no diseñan metodologías para la enseñanza y aprendizaje mediado por las TIC con la participación de los docentes" (Marín et al., 2017, p. 35).

Por su parte, Roux y Roux (2015) destacan la obligación de las instituciones de Educación Superior, de reformar los modelos curriculares de la Arquitectura, atendiendo las exigencias actuales que implican, "la gestión de estudios curriculares bajo paradigmas emergentes que 
justifiquen programas y planes encaminados a la solución de problemáticas globales" (p. 57). Asimismo, se requiere que el estudiante confronte realidades, respondiendo a problemáticas actuales relacionadas con el área en el contexto en que se desenvuelve, preparándose así para la vida laboral, sin estar ajeno al panorama actual del área (Roux y Roux, 2015; León, Eyzaguirre y Gómez, 2016; Dreifuss, 2017; Tobar, 2017; Martínez et al., 2017; Genís-Vinyals, Maroto-Sales \& Taberna-Torres, 2019).

Según estudio realizado por Castellanos, Sierra y Albis (2018), en la Región Caribe Colombiana, se ofrece arquitectura en 9 instituciones universitarias, de las cuales 8 son privadas y una pública. Los enfoques educativos en las universidades de la Región Caribe, han sido pensados desde los modelos tradicionalistas, encontrando dificultades por la restricción de los recursos donde los docentes no cuentan con los recursos suficientes para garantizar que el estudiante obtenga las competencias tanto para su crecimiento académico como para el campo laboral.

$\mathrm{Al}$ abordar a la carrera Tecnología en Gestión de la Construcción de Proyectos Arquitectónicos impartida en la sede Suán de la Universidad del Atlántico, se observa, que existen falencias en el manejo de las herramientas de ofimáticas, convirtiéndose en una debilidad en los estudiantes a la hora de llevar a cabo sus prácticas profesionales. De igual manera esta preocupación la tienen los egresados del programa que tienen que enfrentar esta necesidad y que en algunos casos se les dificulta adaptarse a las nuevas tendencias y cambios en la aplicación del conocimiento convirtiéndose el manejo de las TIC en un componente imprescindible para todas las áreas del saber.

El objetivo de este artículo, es analizar los procesos de enseñanza que requieren, el uso de las TIC del programa Tecnología en Gestión de la Construcción de Proyectos Arquitectónicos y su incidencia en el desempeño académico y prácticas profesionales de los estudiantes del periodo 2019-2 en la sede Suán de la Universidad del Atlántico. Lo anterior teniendo en cuenta el Proyecto Educativo del Programa (PEP), igualmente el testimonio de docentes, estudiantes de últimos semestres, egresados y empleadores de prácticas profesionales.

\section{Metodología}

La investigación está enmarcada bajo el paradigma hermenéutico, denominado también, cualitativo, fenomenológico y humanista; en aras de entender las dinámicas asociadas al proceso de enseñanza docente en un programa tecnólogo de arquitectura y las repercusiones en el aprendizaje y desempeño laboral de los estudiantes, por medio de la participación de los actores inmersos en la actividad académica y laboral. Por tanto, este estudio, está enmarcado en el enfoque cualitativo, recolectando datos para la interpretación y discusión de los mismos, por medio de la descripción y, recurriendo también a la revisión documental. 
La población a estudiar, son los estudiantes de último semestre, docentes y egresados del programa de Tecnología en Gestión de la Construcción de Proyectos Arquitectónicos dictado por la Universidad del Atlántico (Sede Suán), como también, los empleadores de prácticas profesionales. Es así como la muestra, seleccionada por medio del procedimiento no probabilístico causal, estuvo conformada por 2 individuos por cada rol académico (es decir, 2 estudiantes de último semestre, 2 docentes del programa, 2 egresados y 2 empleadores de prácticas de las empresas con las que se ha establecido convenio de prácticas profesionales), para un total de 8 individuos indagados en el semestre 2019-2. Asimismo, teniendo presente el componente documental del estudio, se evalúa el Proyecto Educativo del Programa (PEP), haciendo énfasis en el perfil del egresado y las competencias específicas del programa.

Del mismo modo, se utilizó como instrumento la entrevista semiestructurada, con un formato específico para cada uno de los roles académicos estudiados; precisando en aspectos como el cumplimiento de las competencias específicas del programa, el proceso de enseñanza docente, el proceso de aprendizaje, el uso de las TIC en la enseñanza/aprendizaje, los conocimientos necesarios para las prácticas profesionales y las necesidades actuales del programa tecnólogo. Los cuestionarios de las entrevistas fueron validados por 3 doctores de la Universidad del Atlántico en el año 2019.

En cuanto a los aspectos legales de la investigación, la muestra estudiada diligenció un for- mato de consentimiento informado, en el cual autorizaron, la utilización de los datos, recibiendo información por parte de los investigadores y relacionada con el estudio y su alcance. Todos estos aspectos, se encuentran establecidos de acuerdo con los lineamientos de la normativa colombiana, especificados en la Ley 1581 (Congreso de la República, 2012).

\section{Resultados}

\section{Aspectos destacados en el PEP del programa con relación al uso de las TIC}

Tras la revisión del Proyecto Educativo del Programa (PEP) de la Tecnología en Gestión de la Construcción de proyectos Arquitectónicos, se establece esta carrera como "un campo auxiliar de la formación profesional universitaria en Arquitectura" (Universidad del Atlántico, 2014); asimismo, su enfoque tiene como finalidad:

(...) formar estudiantes en las teorías y principios de las tecnologías disponibles; en las propiedades y significado de los materiales y la forma cómo influyen en el diseño; en los criterios para la gerencia de obras; en las leyes y normativas vigentes en el país relacionadas con seguridad, salud y confort, requeridas en los procesos de construcción y ocupación de los lugares. (...) desarrollar en el estudiante competencias para el trabajo autónomo y colaborativo en ambientes interdisciplinarios, y para la gerencia de proyectos, que son de mayor complejidad, alcance y nivel de gestión que las que corresponden al programa académico (Universidad del Atlántico, 2014). 
De acuerdo con lo que se aprecia en el PEP, la Universidad del Atlántico ha focalizado su modelo de enseñanza/aprendizaje en la conjunción de lo teórico y lo práctico, haciendo énfasis, en la inmersión del estudiante en las tecnologías que actualmente se utilizan para llevar a cabo las actividades auxiliares de la arquitectura. A su vez, la formación está direccionada hacia la búsqueda de adaptabilidad del estudiante en el campo laboral, en proyectos complejos y de alto impacto en materia de arquitectura.

Por otra parte, las competencias específicas del programa, manifiestan el interés de que el estudiante haga uso de las TIC, el manejo de maquinaria especializada, el diseño de proyectos arquitectónicos, el conocimiento sobre sistemas de programación y la elaboración y presentación de presupuestos. Es así como la competencia específica general está compuesta así:

Supervisar y controlar en la construcción de proyectos urbanos-arquitectónicos, la aplicación y cumplimiento de los procesos técnicos de obras, especialmente en la elaboración de presupuestos, supervisión y control de obras ejecutadas, rendimientos y funcionamiento de equipos especializados (...) fomentar el espíritu crítico, la actitud emprendedora e investigativa y el compromiso con el desarrollo humano (Universidad del Atlántico, 2014).

La Universidad del Atlántico, pretende a través de este programa, la obtención y creación de nuevos empleos, e induce a que el docente, fomente al estudiante hacia la planificación y gestión de proyectos de arquitectura, formulación de propuestas y emprendimiento, la resolución de problemáticas vigentes del contexto en que se desenvuelve asociadas al campo de estudio y el uso de las TIC, siendo este último un aspecto resaltado en el programa tecnólogo.

Es así, como desde el programa tecnólogo, se ha establecido, que "en el desarrollo de las actividades, tanto de docencia, como investigativas, se promueve el uso de las tecnologías de la información y la comunicación" (Universidad del Atlántico, 2014). Son entonces los intereses de la Universidad del Atlántico, desde su programa tecnólogo, fortalecer el uso y apropiación de las TIC, para su utilización en las actividades académicas y al servicio de la actividad laboral del futuro egresado.

Perspectiva docente relacionadas al uso de las TIC en el programa tecnólogo

Cuando se analiza la percepción docente con respecto al uso de las TIC en el programa tecnólogo, es preciso conocer que, al consultar a los docentes con respecto a ¿qué estrategias didácticas basadas en las TIC, utiliza para la enseñanza en el programa tecnólogo?, estos manifiestan lo siguiente:

Para el docente encargado de la materia costos y presupuestos (Docente 1), afirma que, "teniendo en cuenta que la materia de presupuestos exige el uso de herramientas de office como Excel, mis clases no se dan en la sala de informática, por lo cual, los estudiantes no interactúan de manera frecuente con el computador; no obstante, la falta de insumos tecnológicos ha dificultado la realiza- 
ción de las clases y de proponer alternativas desde la educación" (Docente 1, comunicación personal, 11 de diciembre de 2019).

Según el docente de la materia componentes básicos de diseño en contexto (Docente 2), afirma que este no aplica estrategias didácticas basadas en el uso de las tecnologías, puesto que "muchos estudiantes no saben manejar el computador; el teclado ni acceder a los softwares utilizados para la realización de actividades en planos computarizados, por lo cual, he optado por limitar las clases a la enseñanza de conceptos y normativa vigente en arquitectura. Lógicamente no es lo apropiado teniendo en cuenta los intereses del programa, pero, hay además problemas con los computadores, generalmente no contamos con la cantidad de computadores para que todos los estudiantes participen" (Docente 2, Comunicación personal, 11 de diciembre de 2019).

Por su parte, al consultar a los docentes sobre, ¿cómo ha incidido su modelo de enseñanza basado en las TIC en el aprendizaje de los estudiantes?, el Docente 1 manifiesta que, "los estudiantes han aprendido a manejar el Excel el cual es útil no solo para la clase, sino también para sus prácticas profesionales y el campo laboral, además que ya reconocen otras herramientas del paquete de Office" (Docente 1, comunicación personal, 11 de diciembre de 2019); por su parte, para el Docente 2, "hay que ser sinceros. Los estudiantes no han tenido la formación necesaria en materia de uso de herramientas ofimática, $y$, teniendo en cuenta las condiciones actuales en las que se dictan las clases, debo reconocer que los estudiantes pre- sentan falencias importantes en materia del uso de las TIC, que pueden incidir negativamente en su desempeño académico y laboral (Docente 2, comunicación personal, 11 de diciembre de 2019).

Por otra parte, al consultar a los docentes, ¿cree usted que el manejo apropiado de los recursos TIC sirve para el mejoramiento del desempeño en los procesos de enseñanza y aprendizaje en el entorno académico?, el Docente 1, responde así: “considero que las tecnologías son importantes teniendo en cuenta el mundo globalizado, por lo tanto, creo que el manejo de las TIC facilita la enseñanza y el aprendizaje, pero, cuando el estudiante no cuenta con conocimientos básicos en ofimática, es mejor recurrir a otras alternativas de enseñanza" (Docente 1 , comunicación personal, 11 de diciembre de 2019). Por su parte, para el Docente 2, "el uso de las TIC y su manejo apropiado son determinantes en el proceso de enseñanza aprendizaje, es por eso que, considero, que contribuyen no solo al mejoramiento de las dinámicas académicas, sino también, en la preparación para el campo laboral" (Docente 2, comunicación personal, 11 de diciembre de 2019).

Finalmente, Al consultar a los docentes sobre ¿qué opinión le merece la siguiente afirmación?: Una de las características principales que definen las TIC es la adaptabilidad a los procesos de enseñanza y aprendizaje, el Docente 1 responde: "cuando se habla de adaptabilidad se refiere a que el docente debe fomentar en el estudiante el uso de las tecnologías, pero, yo considero que, si el estudiante no sabe manejar el computador, es difícil adaptar estrategias de enseñanza apren- 
dizaje basadas en las tecnologías" (Docente 1, comunicación personal, 11 de diciembre de 2019).

Contrario a este pensamiento, el Docente 2 considera que "es importante que se incluyan las nuevas tecnologías en los procesos de enseñanza aprendizaje, por lo cual, estoy de acuerdo con esta afirmación. Es preciso que, dentro del programa, se incluya la enseñanza del manejo básico del dibujo asistido por computador, y que la ofimática sea paralela con su aprendizaje en los procesos de la arquitectura, y que los docentes debemos llevar a cabo, dinámicas atractivas basadas en el uso de las tecnologías, fomentando el interés del estudiante por el aprendizaje en materia de las TIC" (Docente 2, comunicación personal, 11 de diciembre de 2019).

Percepción de los estudiantes sobre el uso de las TIC

Al consultar si, ¿dentro del programa le brindan cursos para el manejo apropiado de las TIC?, el Estudiante 1, manifestó lo siguiente: "No nos brindan ningún curso. De hecho, es una exigencia que hemos realizado a los encargados del programa y nunca han prestado atención a esto. Hemos hablado con los docentes también, y muchos de ellos manifiestan que eso es importante" (Estudiante 1, comunicación personal, 07 de diciembre de 2019); asimismo, para el Estudiante 2, "no han planteado una materia para que nosotros aprendamos cosas básicas sobre informática, $\mathrm{y}$, algunos profesores nos dicen que nosotros deberíamos tener conocimientos básicos sobre el uso de las tecnologías porque en nuestras prácticas nos van a exigir eso" (Estudiante 2, comunicación personal, 07 de diciembre de 2019).

Por otro lado, al preguntar si, ¿Los docentes aplican actividades para la enseñanza basada en las TIC?, el Estudiante 1 responde que "son muy pocas. Es que vamos muy poco a la sala de informática, porque la mayoría de profesores colocan actividades en el salón y trabajamos con libros y fotocopias" (Estudiante 1, comunicación personal, 07 de diciembre de 2019). Por su parte, para el Estudiante 2, "los docentes no aplican actividades de enseñanza que tengan que ver con tecnologías, ya que hay muy pocos elementos de informática, como ellos mismos lo manifiestan. Nosotros con el profesor trabajamos en la clase con fotocopias y algunos a veces llevan portátiles" (Estudiante 2, comunicación personal, 07 de diciembre de 2019).

Con respecto a la pregunta, ¿Considera que es importante el aprendizaje sobre las TIC dentro del programa?, el Estudiante 1, afirma: "claro que es importante, porque eso facilita que captemos el conocimiento, al igual que nos fortalece para las prácticas profesionales. Otros compañeros que han estado en prácticas me han comentado que uno debe manejar el computador y unos softwares cuando ingresa a una empresa de construcción" (Estudiante 1, comunicación personal, $07 \mathrm{de}$ diciembre de 2019). Para el Estudiante 2, "las TIC son importantes para que uno aprenda a realizar diseños por computador, trabajar en Excel y redactar en Word. Por eso, ojalá se mejoren las salas de informática y que las doten con más computadores" (Estudiante 2, comunicación personal, 07 de diciembre de 2019). 
Finalmente, al cuestionar ¿por qué cree que es importante conocer sobre las TIC cuando ingresa al campo laboral?, el Estudiante 1 respondió: "es importante porque las empresas de construcción que necesitan auxiliares en arquitectura, exigen que uno tenga conocimientos básicos de informática, especialmente de softwares y de Excel, como me han comentado mis amigos que han estado en prácticas y que trabajan en empresas" (Estudiante 1, comunicación personal, 07 de diciembre de 2019). Entre tanto, el Estudiante 2, manifestó lo siguiente: "claro que es importante. Yo lo digo porque a un amigo que egresó del programa lo sacaron de una empresa en la que estaba porque no sabía utilizar las herramientas de informática básica y no manejaba bien el computador. Por eso es que hemos estado exigiendo que nos den un curso sobre informática básica" (Estudiante 2, comunicación personal, 07 de diciembre de 2019).

\section{Percepción del egresado sobre el uso de las TIC en sus prácticas profesionales}

Resaltando la opinión de los egresados, al ser consultados: ¿Cree usted que el conocimiento sobre el uso de las tecnologías modernas es determinante a la hora de desempeñar las prácticas profesionales?, el Egresado 1 respondió: "claro que sí. A mí me costó mucho cuando entré a la empresa hacer mis prácticas porque me mandaban a que hiciera cosas en Excel con fórmulas y yo no sabía, lo mismo cuando me pedían tablas dinámicas y esas cosas" (Egresado 1, comunicación personal, 23 de diciembre de 2019). Por su parte, el Egresado 2, expresó: "son importantes, porque muchos de los empleadores de prácticas en las empresas miran como uno maneja el computador, si uno sabe utilizar softwares de dibujo y esas cosas" (Egresado 2, comunicación personal, 23 de diciembre de 2019).

$\mathrm{Al}$ cuestionar sobre, ¿Qué herramientas relacionadas con las TIC aprendió a manejar usted dentro de su programa tecnólogo?, el Egresado 1 respondió: "a nosotros nos enseñaron sobre Excel, Word y Power Point, pero muy elemental. Teníamos dificultad para acceder a la sala de informática. A nosotros no nos enseñaron a manejar bases de datos ni nada de eso" (Egresado 1, comunicación personal, 23 de diciembre de 2019). Por su parte el Egresado 2 manifestó: "nos enseñaron Office básico, pero eso no fue suficiente para las prácticas. No nos enseñaron herramientas de dibujo ni más nada, y eso fue un problema porque afecta nuestro desempeño laboral" (Egresado 2, comunicación personal, 23 de diciembre de 2019).

Por otro lado, al consultar, en sus prácticas profesionales, ¿le exigieron el manejo de softwares por medio del computador?, el Egresado 1 afirmó que, "si me exigieron el manejo de softwares, pero era una cuestión muy básica. La verdad yo no sabía al principio hacer eso, después, en la empresa, la persona que me dio la inducción me indicó algunas cosas y así pude manejar el software. El problema estuvo cuando me colocaban a que hiciera presupuestos en Excel" (Egresado 1, comunicación personal, 23 de diciembre de 2019). Entre tanto, según el Egresado 2, "si nos exigieron el manejo de softwares. Aunque, a mí 
me colocaron fue a llevar el inventario por medio de un software, muchas veces me tocaba utilizar Excel y para mí era muy difícil, porque tenía uno que hacer tablas dinámicas y fórmulas" (Egresado 2, comunicación personal, 23 de diciembre de 2019).

Finalmente, al consultar a los egresados ¿Cuál fue la mayor dificultad al hacer sus prácticas profesionales?, el Egresado 1 reconoció que "lo más difícil fue el manejo de Excel. Eso si fue una cuestión que me costó muchísimo. Yo no sabía hacer fórmulas, ni nada de eso, y siempre me llamaban la atención en la empresa por los errores en los documentos. Vine a aprender fue cuando terminé mis prácticas" (Egresado 1, comunicación personal, 23 de diciembre de 2019). Por su parte, para el Egresado 2, "lo que más se me dificultó fue hacer planos en el computador. Eso si es difícil. Yo trataba y nada que me salía lo que me pedían, me costó mucho hacer eso, y me llamaron mucho la atención porque no sabía de eso, y ellos me decían que eso era muy básico" (Egresado 2, comunicación personal, 23 de diciembre de 2019).

\section{Percepción de los empleadores de prácticas profesionales}

Por último, los empleadores de las prácticas profesionales que se encuentran en las empresas, al ser consultados, ¿Cuál cree que son las falencias principales en materia de manejo de las tecnologías por parte de practicantes?, el Empleador 1 respondió: "las falencias principales de los practicantes que han venido a la empresa siempre son las mismas, el mal manejo del computador, el desconocimiento de softwares básicos para la digitación de datos y planos, la dificultad para utilizar herramientas básicas como Excel. Algunos practicantes, por pena, no preguntan a otras personas que trabajan con ellos, prefieren hacer las cosas mal que dejarse guiar y esa es otra de las dificultades del programa" (Empleador 1, comunicación personal, 11 de diciembre de 2019). Por su parte, el Empleador 2, manifestó: "los muchachos que han venido a hacer prácticas aquí, no saben manejar ni siquiera Excel básico y se les dificulta hacer presupuestos. Nosotros accedemos a recibirlos para que hagan este tipo de actividades y nos toca reforzar sus conocimientos aquí, lo cual no debería ser" (Empleador 2, comunicación personal, 11 de diciembre de 2019).

Por otro lado, al cuestionar sobre, ¿Qué conocimientos específicos debe tener el practicante de arquitectura en materia de TIC para el desempeño asertivo de sus funciones?, el Empleador 1 afirma que "es preciso que el estudiante sepa manejar el paquete de Microsoft Office, especialmente Excel, como también conocimientos básicos en dibujo asistido por computador. También, el practicante debe conocer sobre cómo llevar inventarios, teniendo en cuenta su labor como auxiliar en arquitectura" (Empleador 1 , comunicación personal, 11 de diciembre de 2019). También, el Empleador 2, reconoce que "los estudiantes, para realizar sus prácticas profesionales asertivamente, requieren de poseer conocimientos en manejo de Excel y Word, que 
son las herramientas que más utilizan para el desarrollo de sus actividades. Yo entiendo que muchos no tengan conocimientos avanzados en materia de estas herramientas, pero a veces, vienen practicantes que no saben ni siquiera cómo prender el computador" (Empleador 2, comunicación personal, 11 de diciembre de 2019).

Finalmente, al cuestionar a los empleadores sobre ¿qué recomendaciones brindaría al programa para el mejoramiento de la formación de los estudiantes?, el Empleador 1 expresa que, "es preciso que el programa induzca al estudiante a que sepa asociar los conocimientos y la práctica, la cual se ve reflejada en sus actividades en la empresa cuando vienen aquí a realizar sus prácticas. Por eso, deben buscar que el estudiante aprenda, sobre el manejo básico del computador, el acceso a herramientas ofimáticas, el aprendizaje al menos básico de bases de datos y que se les fomente hacia el trabajo en equipo, ya que algunos no se dejan guiar" (Empleador 1, comunicación personal, 11 de diciembre de 2019).

Asimismo, el Empleador 2 reconoce que, "es importante que los estudiantes aprendan a manejar herramientas informáticas, indispensables no sólo para sus prácticas, sino también, para su vida laboral y proyectos personales. Por eso, yo creo que los docentes deben enseñar paralelamente la teoría y el manejo de la informática, porque la verdad es que el mundo se mueve a través de las tecnologías y la arquitectura actualmente, requiere de gente con conocimiento en esta materia (Empleador 2, comunicación personal, 11 de diciembre de 2019).

\section{Discusión Y CONCLUSIONES}

Los procesos de enseñanza del programa Tecnología en Gestión de la Construcción de Proyectos Arquitectónicos de la Universidad del Atlántico sede Suán, que requieren la utilización de las TIC, no han sido promovidos para ser impartidos de carácter obligatorio y contribuir a mejorar la calidad de la educación, tal y como se aprecia en la información obtenida por parte de los docentes, ya que estos concuerdan, en las dificultades que han encontrado y que persisten en materia de insumos tecnológicos, representando un obstáculo, para inducir al estudiante al uso de las tecnologías como una herramienta de aprendizaje. Por tanto, el docente no posibilita esta premisa de la Educación Superior; la cual tiene la obligación de brindar posibilidades y herramientas al estudiante para el desempeño de su profesión durante toda la vida (Pimienta, 2012; Guevara, 2013; Castillo \& Del Castillo, 2015; Núñez, 2016; Hernández-Rojas et al., 2017; Hernández, 2017; Pérez et al., 2017).

Los objetivos trazados en el Proyecto Educativo del Programa (PEP), están distantes de los intereses del profesorado, estudiantes y empleadores de prácticas, teniendo presente que, a pesar de que el enfoque del programa tecnólogo invita a que el estudiante sea instruido en materia del uso de las tecnologías, los testimonios de los actores participantes dan cuenta del incumplimiento de las condiciones mínimas para que los estudiantes puedan adquirir las competencias específicas, para conseguir un desempeño asertivo a nivel 
académico, en sus prácticas profesionales y a nivel laboral. Estos resultados dan cuenta, de lo manifestado por Marín et al. (2017), quienes afirman, que las instituciones de Educación Superior, a pesar de reconocer la relevancia del uso de las TIC en la formación, - en su mayoría - no disponen de cursos para que docentes y estudiantes se capaciten en las TIC de manera continua.

Por otro lado, al observar la relación existente entre los procesos de enseñanza pensados por el docente y las formas de aprendizaje del estudiantado referentes al uso de las tecnologías, se aprecian ciertas dificultades, teniendo presente, el desconocimiento por parte de los estudiantes, sobre el manejo de herramientas ofimática en niveles básicos y las alternativas brindadas por los docentes, para llevar a cabo sus actividades académicas con respecto al uso de computadores. Sin embargo, desde el programa se piensa en la relevancia de asociar lo teórico con lo práctico; la realidad da cuenta, de problemáticas asociadas a la falta de recursos tecnológicos y la conservación de modelos de enseñanza tradicionalistas. Esto concuerda con la investigación realizada por Castellanos, Sierra y Albis (2018), donde se evidenció, que los enfoques educativos en las instituciones de Educación Superior de la Región Caribe colombiana, en materia de arquitectura, han sido pensados desde los modelos tradicionalistas de enseñanza/aprendizaje, sin contar los docentes, con recursos suficientes para garantizar que el estudiante adopte competencias, tanto para su crecimiento académico como para su desempeño laboral, al igual que sucede también en otros países Latinoamericanos (Romero, 2016; Botasso \& Tanoune, 2016).

En la mayoría de los casos, la poca interacción con las TIC ha conllevado a que los estudiantes en sus prácticas profesionales, no se adapten a las dinámicas laborales en las que se requiere el uso de herramientas tecnológicas en nivel básico y softwares para la digitación de planos y datos, generando inconformismo en los empleadores de las prácticas profesionales, teniendo presente que, desde la formación del programa, deberían brindarse las capacitaciones en materia de uso de herramientas como Microsoft Office y dibujo asistido por computador para llevar a cabo el rol de auxiliar en arquitectura, perfil establecido en el PEP del programa y que resulta ser una competencia específica.

Las regiones del caribe colombiano necesitan que el sistema educativo actual alcance el liderazgo en la aplicación de los sistemas TIC, dando versatilidad a los egresados, fortaleciendo su campo laboral en diversos sistemas aplicables a la arquitectura. Se mencionan sistemas, que los estudiantes deben conocer, dominar y saber aplicar: Los sistemas de información geográfica, utilizados en proyectos urbanos, territoriales, topografía y ejercicios afines. Por esta razón es imprescindible contar con la infraestructura apropiada y el material tecnológico y en permanente actualización. Requerir que los docentes pertenecientes a los programas mencionados, se encuentren calificados en TIC, constituyéndose un equipo preparado para enfrentar los desafíos del nuevo milenio. 
Lo antes mencionado se asocia con los resultados obtenidos en diversas investigaciones, donde se apreció, que en materia TICS, el programa estudiado se encuentra distante a la realidad educativa, llegando a la conclusión que, para garantizar que el estudiante, cuente con todos los componentes educativos de manera asertiva en su inmersión en el ámbito laboral, debe conocer estrategias que lo ayuden a comprender y resolver problemas dentro de su contexto, haciendo uso de los recursos - como las tecnologías- propios del mundo globalizado (Vélez, 2011; Correal y Verdugo, 2011; Villar, 2012 citado en Guevara, 2013; Erazo y Sánchez, 2013; Rueda, 2014; Díaz, 2016; Gasco y Melo, 2017; O’Byrne y Quintana, 2018; Melo, 2018; Perlaza y Betancourt, 2018; Martínez y García, 2019; González et al., 2019).

Por último, de acuerdo con los resultados obtenidos, es preciso que los docentes adapten a su proceso de enseñanza el uso de las nuevas tecnologías, considerando la asociación entre lo teórico y lo práctico, que abarque no solo la educación, sino también, pensando en el desempeño laboral. También, es preciso que las Instituciones de Educación Superior garanticen el acceso y la capacitación del docente en materia de las TIC, para ser utilizadas como un recurso para la enseñanza. Asimismo, debe incluirse al estudiante en el proceso de enseñanza, teniendo en cuenta su capacidad creativa, innovadora y emprendedora, fomentando a la búsqueda de soluciones asertivas a las situaciones presentes en su contexto en materia de arquitectura, $y$, de acuerdo a las tendencias actuales dadas a partir de la globalización.
Las reformas curriculares se hacen imprescindibles, los ritmos de aprendizaje deben ser personalizados y proporcionar los elementos necesarios, para que pueda existir la autorregulación, la autonomía y la adaptación a nuevas formas de aprender y comunicarse; requiriendo involucrar a la comunidad académica en la transformación curricular y en el conocimiento de la propuesta. Igualmente, con la retroalimentación de lo propuesto, crear en consenso un plan de mejoras.

Integrar las TIC's en las Instituciones de Educación Superior, integrando y valorando la participación de la comunidad académica, mejorando y transformando dichas prácticas educativas, y de igual forma, reforzar el aprendizaje auto dirigido y autónomo. Sobre esto, Rocha (2015) asevera que, "el trabajo autónomo, está considerado como una opción poderosa y eficaz, que sobrevive a cualquier modelo productor de enseñanza" (pp. 44-45). Lo anterior con el fin de que los estudiantes tengan dominio sobre sus procesos en la adquisición del conocimiento utilizando los medios apropiados y eficientes para su realización. La cuestión curricular Currículo ideal-, debe estar integrado a la práctica - Currículo en contexto-; acompañado de una transformación mental, y que, a su vez, responda a la demanda en el campo profesional-laboral.

Todo lo anterior, con el fin de promover escenarios innovadores como lo mencionan Newmann y Kyriakakis (2004), citado en Andrade, Covelo y Vega (2010), ofreciendo sistemas de inmersión 
remota, experiencias interactivas, ambientes dinámicos, utilización de dispositivos especiales, material multimedia, uso inteligente del internet entre otros. Igualmente, se piden habilidades por parte de la comunidad educativa, Monereo y Pozo (2008), quienes solicitan que sean los gobiernos quienes den el apoyo a través de fondos provenientes del ministerio de las TIC's, fortalecerlas y transformar de esa manera los procesos cognitivos y la práctica de ese mismo conocimiento de las Instituciones de Educación Superior. Se requiere también la alfabetización digital permanente por lo cambiante que resultan estos sistemas, y que los currículos puedan organizarse y funcionar en distintos ambientes abiertos de aprendizaje. El reto comienza y es tarea de todos, especialmente en épocas de pandemia como la que afrontamos actualmente.

\section{REFERENCIAS}

Andrade, M., Covelo, E. y Vega, F. (2010). Ventajas del uso de las TIC en la enseñanza/ aprendizaje de la materia Contaminación de Suelos. Revista de Ciencias Agrarias, 33(1), 257-266. https://doi.org/10.19084/ rca. 15823

Botasso, G. \& Tanoue, S. (2016). Construindo Olhares: o Desenho nas Obras de Eduardo Souto de Moura. Cadernos de Pós-Graduação em Arquitetura e Urbanismo, 15(2), 131-151. http://editorarevistas.mackenzie.br/index. php/cpgau/article/view/2015.2.Botasso
Castellanos, A., Sierra, A. y Albis, C. (2018). Panorama actual de los programas de arquitectura en Colombia. En, G. Martínez y M. Albis (Edits.), Enfoques, Teorías y Perspectivas de la Arquitectura y sus Programas Académicos (pp. 139-172). Sincelejo: CECAR. Recuperado de https://www.cecar.edu.co/documentos/ editorial/e-book/enfoques-teorias-y-perspectivas-de-la-arquitectura-y-sus-programasacademicos.pdf

Castillo, C. y Del Castillo, M. (2015). Enseñenaza, sustentabilidad, arquitectura. Arquitecturas del Sur, 33(48), 30-43. Disponible en http://revistas.ubiobio.cl/index.php/AS/ article/view/1952

Cohen, E. y Correa, J. (2018). El programa de arquitectura en el contexto institucional. En, G. Matínez y M. Albis (Edits.), Enfoques, Teorias y Perspectivas de la Arquitectura y sus Programas Académicos (pp. 173-190). Sincelejo: CECAR. Recuperado de https://www. cecar.edu.co/documentos/editorial/e-book/ enfoques-teorias-y-perspectivas-de-la-arquitectura-y-sus-programas-academicos.pdf

Correal, G. y Verdugo, H. (2011). Sobre modelos pedagógicos y el aprendizaje del proyecto arquitectónico. Revista de Arquitectura, 13(1), 80-91. Disponible en https://revistadearquitectura.ucatolica.edu.co/article/view/772

Díaz, L. (2016). Plan para la formación curricular de cátedras de accesibilidad en las facultades de Arquitectura en Colombia. Espiral, 6(1), 91-106. https://doi.org/10.15332/erdi. v6i1.1589 
Dreifuss-Serrano, C. (2017). Docencia en arquitectura: claves de lectura. Pedagogía y Arquitectura, 1(1), 9-17. https://doi.org/10.31381/ pedagogiaarquitectura.v0i1.765

Erazo, E. y Sánchez, P. (2013). Incidencia de los medios de expresión digital en formación de arquitectos y arquitectas. RLCSNJ, 11(2), 769-81. Disponible en http://revistaumanizales.cinde.org.co/rlcsnj/index.php/RevistaLatinoamericana/article/view/950

Gasco, J. y Melo, M. (2017). Importancia de la selección de recursos de Tecnología, Información y Comunicación (TIC) en la educación superior en las universidades de Colombia. En, R. Roig-Vila (Ed.), Investigación en docencia universitaria. Diseñando el futuro a partir de la innovación educativa (pp. 528-537). Barcelona: Octaedro. Recuperado de https:// rua.ua.es/dspace/bitstream/10045/71157/1/ Investigacion-en-docencia-universitaria_55. pdf

Genís-Vinyals, M., Maroto-Sales, J. y TabernaTorres, J. (2019). La influencia del espacio en el aprendizaje de la arquitectura. Bauhaus de Dessau y ENSA Nantes. Zarch, (12), 82-95. https://doi.org/10.26754/ojs_zarch/ zarch.2019123544

González, M., Portero, A., Machado, R. y Fariñas, M. (2018). Dos asignaturas en un solo proceso de enseñanza. Innovación pedagógica en la formación de arquitectos. Apuntes, 31(2), 1-9. https://doi.org/10.11144/Javeriana.apu31-2.apei
Guevara, O. (2013). Análisis del proceso de enseñanza aprendizaje de la Disciplina Proyecto Arquitectónico, en la carrera de Arquitectura, en el contexto del aula. [Tesis Doctoral]. Universitat Autónoma de Barcelona, Barcelona, España. Disponible en https://www. tdx.cat/handle/10803/116191

Hernández, S. (2017). La sustentabilidad en la enseñanza de la arquitectura en México. La Colmena, (59), 133-135. Disponible en https://lacolmena.uaemex.mx/article/ view/5989

Hernández-Rojas, L., Suárez-Castrillón, S. y Rico-Bautista, D. (2017). La gamificación y arquitectura funcional: estrategia práctica en el proceso de enseñanza/aprendizaje usando la tecnología. Ingenio UFPSO, 14(1), 123136. https://doi.org/10.22463/2011642X.2201

León, N., Eyzaguirre, S. y Gómez, J. (2016). Arquitectura de software Cultiventura, herramienta de soporte a la enseñanza-aprendizaje de la cultura Moche "videojuegos y realidad humana”. Campus, 21(21), 91-100. Disponible en https://www.aulavirtualusmp. pe/ojs/index.php/rc/article/view/1140

León, A., Risco, E. y Alarcón, C. (2014). Estrategias de aprendizaje en educación superior en un modelo curricular por competencias. Revista de la Educación Superior, 43(4), 123-144. Disponible en http://publicaciones. anuies.mx/revista/172/3/6/es/estrategias-deaprendizaje-en-educacion-superior-en-unmodelo 
Marín, F., Inciarte, A., Hernández, H. y Pitre, R. (2017). Estrategias de las Instituciones de Educación Superior para la Integración de las Tecnología de la Información y la Comunicación y de la Innovación en los Procesos de Enseñanza. Un Estudio en el Distrito de Barranquilla, Colombia. Formación Universitaria, 10(6), 29-38. http://dx.doi. org/10.4067/S0718-50062017000600004

Márquez, J. (2017). Tecnologías emergentes, reto para la educación superior colombiana. Ingeniare, (23), 35-57. https://doi. org/10.18041/1909-2458/ingeniare.2.2882

Martin, J. (2018). Calidad educativa en la educación superior colombiana. una aproximación teórica. Sophia, 14(2), 4-14. http:// dx.doi.org/10.18634/sophiaj.14v.2i.799

Martínez, J., Tobón, S. y Romero, A. (2017). Problemáticas relacionadas con la acreditación de la calidad de la educación superior en América Latina. Innovación Educativa, 17(73), 79-96. http://www.scielo.org.mx/pdf/ ie/v17n73/1665-2673-ie-17-73-00079.pdf

Martínez, P. y García, E. (2019). Extensión y práctica pedagógica en arquitectura. Estoa, 8(15), 147-156. https://doi.org/10.18537/est. v008.n015.a12

Melo, M. (2018). La integración de las TIC como vía para optimizar el proceso de enseñanzaaprendizaje en la Educación Superior en Colombia. [Tesis Doctoral]. Universidad de Alicante, Alicante, España. https:/rua. ua.es/dspace/bitstream/10045/80508/1/tesis_myriam_melo_hernandez.pdf
Monereo, C. y Pozo, J. (2008). El alumno en entornos virtuales: condiciones, perfiles y competencias. En, C. Coll y C. Monereo (Eds.), Psicología de la Educación Virtual (pp. 109-130). Madrid: Morata.

Núñez, J. (2016). El modelo competencial y la competencia comunicativa en la Educación Superior en América Latina. Foro de Educación, 14(20), 467-488. http://dx.doi. org/10.14516/fde.2016.014.020.023

O’Byrne, M. y Quintana, I. (2018). La historia en la formación del arquitecto o la dicotomía entre el hacer y el saber. Dearq, (22), 8-11. https://doi.org/10.18389/ dearq22.2018.00

Pérez, J., Daza, M. \& Orozco, E. (2017). Estrategias de enseñanza aprendizaje para el fomento del pensamiento crítico en la Educación Superior. Gestión, competitividad e innovación, 5(2), 62-71.

Perlaza, P. y Betancourt, W. (2018). Formación integral en arquitectura: Hacia una pedagogía innovadora / Integreal training (Education) in Architecture Towards an Innovative Pedagogy. Revista Internacional de Educación y Aprendizaje, 6(2), 69-72. https://doi.org/10.37467/gka-revedu. v6.1457

Pimienta, J. (2012). Estrategias de enseñanza aprendizaje: docencia universitaria basada en competencias. Naucalpan de Juárez: Pearson. 
República de Colombia. Congreso de la República. (18 de octubre de 2012). Por la cual se dictan disposiciones generales para la protección de datos personales. [Ley 1581]. Diario Oficial: No. 48.587. Disponible en http://www.secretariasenado.gov.co/senado/ basedoc/ley_1581_2012.html

Rocha, D. (2015). La enseñanza y la Educación autónoma. Revista Amauta, (26), 41-47. Recuperado de http://investigaciones.uniatlantico.edu.co/revistas/index.php/Amauta/ article/view/1318/967

Romero, M. (2016). Fundamentos de la creación de la nueva carrera de Arquitectura, Urbanismo y Territorio e implementación pedagógica con mención en Arquitectura en una universidad privada de Lima. [Tesis de Mestría]. Universidad San Ignacio de Loyola, Lima, Perú. Recuperado de http://repositorio.usil.edu.pe/bitstream/ USIL/2716/1/2017_Romero_Fundamentos-de-la-creacion-de-la-nueva-carrera. pdf

Roux, D. y Roux, R. (2016). Justificación curricular de la asignatura Arquitectura de tierra. Caso: Tamaulipas, México. Educación y Humanismo, 18(30), 57-70. http://dx.doi. org/10.17081/eduhum.18.30.1322

Rueda, C. (2014). Cuestiones de método creativo. Metamorfosis y conciencia material en los procesos creativos en arquitectura. Revista de Arquitectura, 16, 58-67. http:// dx.doi.org/10.14718/RevArq.2014.16.7
Ruiz, H. y Duarte, F. (2017). Un ejercicio de ruptura en la construcción del currículo en la Educación Superior. Caso Universidad Piloto de Colombia. Uni-pluri/versidad, 17(2), 88-101. Recuperado de https://revistas.udea.edu.co/index.php/unip/article/ view/334015/20789982

Tobar, A. (2017). Índice de las competencias TIC en docentes de Educación Superior. Campus Virtuales, 6(2), 113-125. Disponible en http://www.uajournals.com/ojs/index.php/ campusvirtuales/article/view/240

Universidad del Atlántico. (2014). Programa académico de la Tecnología en Gestión de la Construcción de Proyectos Arquitectónicos. [PEI Institucional]. Universidad del Atlántico, Barranquilla, Colombia.

Vélez, S. (2011). La experiencia de la ENHSA: ¿cuál será el futuro en la formación de los arquitectos? Revista Uniandes, (9), 18-29. https://doi.org/10.18389/dearq9.2011.04

Villar, M. (2012). Estrategia didáctica para el aprendizaje de la historia y la teoría de la arquitectura. Revista de Arquitectura, (13), 76-85. Disponible en https://revistadearquitectura.ucatolica.edu.co/article/ view/729

Cesar Martínez García es Maestrante en Educación Universidad del Atlántico. Arquitecto Universidad del Atlántico. Docente Universidad del Atlántico (Barranquilla, Colombia). https:// orcid.org/0000-0001-9419-4990 
Christiam Castro Escalante es Maestrante en Educación, Universidad del Atlántico. Arquitecto, Universidad del Atlántico. Docente Universidad del Atlántico (Barranquilla, Colombia). https:// orcid.org/0000-0001-9958-8622

Delma Esther Rocha Álvarez es Doctoranda en Educación, Universidad Alas Peruanas. Arquitecta, Corporación Universitaria de la Costa (CUC). Docente Universidad del Atlántico (Barranquilla, Colombia). http://orcid.org/00000001-8592-2554

Isaac Nieto Mendoza es Filósofo, Universidad del Atlántico (Barranquilla, Colombia). https:// orcid.org/0000-0001-5302-6931 